\title{
Innovative biodegradable poly(L-lactide)/ collagen/hydroxyapatite composite fibrous scaffolds promote osteoblastic proliferation and differentiation
}

\author{
This article was published in the following Dove Press journal: \\ International Journal of Nanomedicine \\ 13 October 2017 \\ Number of times this article has been viewed
}

\author{
Guoqiang Zhou ${ }^{1-3}$ \\ Sudan Liu' \\ Yanyan Ma' \\ Wenshi $\mathrm{Xu}{ }^{\prime}$ \\ Wei Meng' \\ Xue Lin' \\ Wenying Wang ${ }^{1,3}$ \\ Shuxiang Wang ${ }^{1-3}$ \\ Jinchao Zhang ${ }^{1-3}$ \\ 'College of Chemistry and \\ Environmental Science, ${ }^{2} \mathrm{Key}$ \\ Laboratory of Medicinal Chemistry \\ and Molecular Diagnosis of Ministry \\ of Education, ${ }^{3}$ Key Laboratory of \\ Chemical Biology of Hebei Province, \\ Hebei University, Baoding, Hebei, \\ People's Republic of China
}

\begin{abstract}
The development of an artificial bone graft which can promote the regeneration of fractures or diseased bones is currently the most challenging aspect in bone tissue engineering. To achieve the purpose of promoting bone proliferation and differentiation, the artificial graft needs have a similar structure and composition of extracellular matrix. One-step electrospinning method of biocomposite nanofibers containing hydroxyapatite (HA) nanoparticles and collagen (Coll) were developed for potential application in bone tissue engineering. Nanocomposite scaffolds of poly(L-lactide) (PLLA), PLLA/HA, PLLA/Coll, and PLLA/Coll/HA were fabricated by electrospinning. The morphology, diameter, elements, hydrophilicity, and biodegradability of the composite scaffolds have been investigated. The biocompatibility of different nanocomposite scaffolds was assessed using mouse osteoblasts MC3T3-E1 in vitro, and the proliferation, differentiation, and mineralization of cells on different nanofibrous scaffolds were investigated. The results showed that PLLA/Coll/HA nanofiber scaffolds enhanced cell adhesion, spreading, proliferation, differentiation, mineralization, and gene expression of osteogenic markers compared to other scaffolds. In addition, the nanofibrous scaffolds maintained a stable composition at the beginning of the degradation period and morphology wastage and weight loss were observed when incubated for up to 80 days in physiological simulated conditions. The PLLA/Coll/HA composite nanofibrous scaffolds could be a potential material for guided bone regeneration.
\end{abstract}

Keywords: nanofibrous scaffolds, hydroxyapatite, electrospinning, tissue engineering, degradation, differentiation

\section{Introduction}

Bone defect is a common disease in orthopedic surgery and is difficult to repair. There are several disadvantages associated with the effective repair of bone defects by traditional therapeutic methods such as autograft and allograft, especially in repairing the big bone fracture defects. ${ }^{1}$ The main drawback of autograft is donor shortage. For allograft, the problem is the potential risk of infecting diseases and immunological response. ${ }^{2}$ Titanium alloy, as a bone substitute material, requires reoperation to remove it from the injured part due to difficulty in the degradation process and is usually associated with pain. ${ }^{3,4}$ Therefore, it is necessary to develop a biocompatible material for use in bone regeneration. The scaffold materials are usually used as an initial structure supporting cell growth and phenotype maintenance in bone restoration. ${ }^{5}$ For use in tissue engineering, the ideal scaffold materials should be biocompatible, degradable, and have good mechanical properties. In addition, the materials should have similar structure and
Correspondence: Guoqiang Zhou; Jinchao Zhang

College of Chemistry and Environmental Science, Hebei University, Number 180 Wusidong Road, Baoding, Hebei, 071002, People's Republic of China Email zhougq1982@I63.com; jczhang6970@I63.com BV $\mathrm{NC}$
hereby accept the Terms. Non-commercial uses of the work are permitted without any further permission from Dove Medical Press Limited, provided the work is properly attributed. For permission hereby accept the Terms. Non-commercial uses of the work are permitted without any further permission from Dove Medicat 
composition of extracellular matrices (ECMs) of natural bone tissue. ${ }^{6,7}$ The electrospinning is a favorable and commonly used method for producing materials with similar composition of ECM. ${ }^{8}$ With a high surface area to volume ratio and high porosity, the scaffolds can provide a microenvironment for cell adhesion and growth. ${ }^{9}$ So the electrospun scaffolds are always designed to serve as an ideal bone substitute. ${ }^{10}$

Various synthetic and natural polymers or inorganic substances have been used to fabricate nanofibrous electrospun scaffolds. Ceramics such as bioglass are used for bone regeneration due to their bioactivity and osteoconductive properties. ${ }^{11,12}$ These materials are excellent as an implant to repair bone defect for their similar physical and chemical properties compared with bone mineral components, but they have some problems in terms of fracture and fragile. The polymer materials have good physical properties of toughness. Therefore, scaffold-based polymer such as polycaprolactone (PCL) or polylactic acid (PLLA) were investigated for bone tissue engineering in recent years. ${ }^{13-15}$ In vitro studies showed that a membrane system-based PCL can promote proliferation and early cell differentiation of osteoblast-like cells. ${ }^{15}$ However, the disadvantage of the synthetic polymers is that it has a poor biocompatibility. The degradation products released in the acidic environment may change the physiological environment and affect the functions of cells. ${ }^{16}$ For example, PCL cannot repair bone defect ideally because it gets degraded very rapidly. ${ }^{14}$ Hydroxyapatite (HA) has been used for bone regeneration due to its bioactivity, biodegradability, and osteoconductive properties. ${ }^{17}$ Therefore, biocomposite scaffolds containing HA have been fabricated in previous studies. ${ }^{18,19}$ Two biocompatible, poly(L-lactic acid) (PLLA) and PCL, nanofibrous scaffolds containing HA were synthesized and the effects of scaffolds on the proliferation and differentiation of MC3T3-E1 cells were investigated. The results showed that cells cultured on the composite scaffolds developed actively and the PLLA/PCL/HA scaffolds promoted the differentiation of cells significantly than the PLLA/PCL scaffolds. Due to the low immunogenic response, PLLA has been used in electrospinning techniques as the fundamental materials for synthesis of scaffolds. It has been reported that PLLA-based scaffolds with HA coated on the surface of nanofibers enhanced the osteogenic differentiation of unrestricted somatic stem cells. ${ }^{20}$ Collagen (Coll), as another major component of bone ECM, can enhance cell adhesion, proliferation, and differentiation. The Coll/HA composites not only showed a favorable biocompatibility but also enhanced the bone marrow stromal cell adhesion and differentiation. In addition, Coll/HA scaffolds exhibited no aberrant events complicating the regenerative process. ${ }^{21}$
In this study, HA nanoparticles were prepared by sol-gel method. Then, four composite scaffolds of PLLA, PLLA/HA, PLLA/Coll, and PLLA/Coll/HA were prepared via one-step electrospinning method. The morphology and size of HA nanoparticles and electrospun scaffolds were observed using a transmission electron microscope (TEM) and field emission scanning electron microscope (FE-SEM). The other physicochemical properties of electrospun scaffolds were characterized by energy-dispersive X-ray spectroscopy (EDS), Fourier transform infrared spectroscopy (FTIR), tensile analysis, and water contact angle (WCA) measurements method. The in vitro degradation of scaffolds was investigated. The potential effects of these electrospinning scaffolds on proliferation and differentiation of osteoblasts were evaluated using MC3T3-E1 cells in vitro. Thus, cell viability and cytoskeleton were measured to reflect the biocompatibility of electrospinning scaffolds on cells. In order to elucidate the effects of scaffolds on the differentiation of osteoblasts, alkaline phosphatase (ALP) activity, mineralized matrix formation, and gene expression of cells on the scaffolds were detected. The results may provide useful information for tissue engineering applications of PLLA/Coll/HA nanofibrous scaffolds in the future.

\section{Materials and methods Reagents and materials}

Calcium nitrate tetrahydrate $\left(\mathrm{Ca}\left(\mathrm{NO}_{3}\right)_{3} \cdot 4 \mathrm{H}_{2} \mathrm{O}\right)$, diammonium hydrogen phosphate $\left(\left(\mathrm{NH}_{4}\right)_{2} \mathrm{HPO}_{4}\right)$, ammonia solution $\left(\mathrm{NH}_{3} \cdot \mathrm{H}_{2} \mathrm{O}\right)$, and anhydrous ethanol were purchased from Kemiou Chemical Reagent Co. Ltd. (Tianjin, People's Republic of China). PLLA, molecular weight 100,000 Da, was purchased from Daigang Biotechnology Co. Ltd. (Jinan, People's Republic of China). 3-(4,5-dimethylthiazol-2-yl)-2, 5-diphenyltetrazolium bromide (MTT), dimethyl sulfoxide (DMSO), dexamethasone, bovine tendon collagen, ascorbic acid, insulin, alizarin red-S (ARS), and cetylpyridium chloride were from Sigma-Aldrich Co. (St Louis, MO, USA). 1,1,1,3,3,3-Hexafluoro-2-propanol (HFIP) was obtained from Aladdin Chemical Reagent Co. Ltd. (Shanghai, People's Republic of China). Dulbecco's Modified Eagle's Medium (DMEM), fetal bovine serum (FBS), and trypsin were purchased from Thermo Fisher Scientific, Waltham, MA, USA. 4-Diamidino-2-phenylindole (DAPI) and actin green were obtained from Thermo Fisher Scientific. An ALP activity kit was obtained from the Nanjing Jiancheng Biological Engineering Institute (Jiangsu, People's Republic of China). A micro-protein assay kit was purchased from Beyotime Biotechnology (Jiangsu, People's Republic of China). The mouse osteoblast precursor MC3T3-E1 cell lines were purchased from 
China Infrastructure of Cell Line Resources (Beijing, People's Republic of China). All chemicals used in this experiment were of analytical grade and used without further purification.

\section{Synthesis of hydroxyapatite nanoparticles}

HA nanoparticles were prepared by an optimized sol-gel method. About $2.0 \mathrm{mmol}$ of $\mathrm{Ca}\left(\mathrm{NO}_{3}\right)_{3} \cdot 4 \mathrm{H}_{2} \mathrm{O}$ with certain amount of $\mathrm{NH}_{3} \cdot \mathrm{H}_{2} \mathrm{O}$, to adjust the $\mathrm{pH}$ value (10.0), were dissolved in $20 \mathrm{~mL}$ of anhydrous ethanol which contains polyethylene glycol to form solution 1. Then, $1.2 \mathrm{mmol}$ of $\left(\mathrm{NH}_{4}\right)_{2} \mathrm{HPO}_{4}$ was added into $20 \mathrm{~mL} \mathrm{H}_{2} \mathrm{O}$ to form solution 2. Solution 2 was introduced into solution 1; after vigorously stirring for $2 \mathrm{~h}$ at $25^{\circ} \mathrm{C}$, the obtained collosol was transferred into a water bath kettle and maintained at $60^{\circ} \mathrm{C}$ for $24 \mathrm{~h}$. The precipitates were separated by centrifugation, washed with deionized water and ethanol in sequence, and then dried in air at $100^{\circ} \mathrm{C}$ to obtain the intermediate products. The obtained products were heat treated at $800^{\circ} \mathrm{C}$ for $2 \mathrm{~h}$ to obtain the HA nanoparticles.

\section{Preparation of electrospun scaffolds}

Electrospun nanofibrous scaffolds were prepared via process previously reported with minor changes. ${ }^{1}$ PLLA was dissolved in HFIP to obtain a $54 \%(\mathrm{w} / \mathrm{v})$ solution and then prepared at a high voltage of $15 \mathrm{kV}$ to obtain PLLA scaffolds. PLLA and HA nanoparticles were mixed at a weight ratio of 54:10; PLLA and collagen at a weight ratio of 54:36; and PLLA, collagen, and HA nanoparticles at a weight ratio of 54:36:10 in HFIP to obtain PLLA/HA, PLLA/Coll, and PLLA/Coll/ HA scaffolds, respectively. The solutions obtained were separately loaded in $10 \mathrm{~mL}$ syringes. The distance between spinneret and plate was $15 \mathrm{~cm}$ and a high voltage of $15 \mathrm{kV}$ was applied to prepare these nanofiber scaffolds. The flow rate was set at $0.75 \mathrm{~mL} \cdot \mathrm{h}^{-1}$. The electrospun nanofibers were dried under vacuum for 6 days to remove any residual HFIP.

\section{Characterization techniques}

The morphology and size of HA nanoparticles and electrospun scaffolds were observed using a TEM (Tecnai G2 F20; FEI, Hillsboro, OR, USA) and FE-SEM (JSM-7500F; JEOL, Tokyo, Japan). The diameters of nanofibers were measured from the SEM images using Nano Measurer software (version 1.2). The crystal structure of HA nanoparticles was analyzed by X-ray diffraction (XRD) using monochromic $\mathrm{Cu} \mathrm{K} \alpha$ radiation (D8 Advance; Bruker, Karlsruhe, Germany). The $2 \theta$ angle was varied from $10^{\circ}$ to $80^{\circ}$. The size distribution of HA nanoparticles in medium was evaluated by dynamic light scattering (DLS; Delsa Nano C; Beckman Coulter, Brea, CA, USA). The elements of electrospun scaffolds were analyzed by EDS (PhenomProX; Phenom-World BV, Eindhoven, the Netherlands). The samples were cut into appropriate size and adhered to double-sided adhesive tape, and then some points were chosen to detect the elements of nanofiber scaffolds. FTIR spectra of nanofibrous scaffolds were measured on a spectrometer (Nicolet iS10; Thermo Fisher Scientific). The tensile properties of the electrospun scaffolds were determined by a material testing machine (HZ-1007C; Heng Zhun, Shanghai, People's Republic of China). The hydrophilicity of electrospun scaffolds was investigated using WCA measurements (OCA 15EC; DataPhysics, Filderstadt, Germany). A drop of water dripped from syringe on the nanofiber scaffolds and adhered to the glass slide, and then the contact angle was detected at different time points.

\section{Degradation of scaffolds in vitro}

The degradation measurement of scaffolds was evaluated for up to 80 days in vitro. Four composite scaffolds with an initial individual weight of $10 \mathrm{mg}$ were suspended in individual centrifuge tubes containing $20 \mathrm{~mL}$ of simulated body fluid (SBF) at $\mathrm{pH}$ 7.4. The tubes were sealed and kept in a shaking table which was maintained at $37^{\circ} \mathrm{C}$. At $10,30,50$, and 80 days, samples were rinsed in deionized water and dried in a freeze-dryer. The mass loss percentage of samples was calculated. The morphology of scaffolds after in vitro degradation at different time points was investigated using SEM.

\section{Cell viability assay}

MC3T3-E1 cells were cultured in DMEM supplemented with $10 \% \mathrm{FBS}$, penicillin $(100 \mathrm{U} / \mathrm{mL})$, and streptomycin sulfate $(100 \mathrm{U} / \mathrm{mL})$ in a humidified atmosphere of $5 \% \mathrm{CO}_{2}$ in air at $37^{\circ} \mathrm{C}$ (Model MCO-18AIC; Sanyo, Osaka, Japan). Cells were fed with fresh media every 2 days until the cells reached near confluence $(75 \%-85 \%)$ and trypsinized using trypsin for the next passage. For cell seeding, nanofiber scaffolds were sterilized under UV light for $2 \mathrm{~h}$ on each side and washed with PBS for three times. The cell proliferation was evaluated according to the MTT method. For experiments, cells were seeded on each film at a density of $2 \times 10^{4}$ cells/well in 96-well tissue culture plates and incubated for 1, 3, and 7 days. After that, MTT dye solution $(100 \mu \mathrm{L}, 5 \mathrm{mg} / \mathrm{mL})$ was added to each well at the predetermined time point. MTT was aspirated and $200 \mu \mathrm{L}$ of DMSO was added after $4 \mathrm{~h}$ of cultivation. Then, $100 \mu \mathrm{L}$ of the solution was added to another 96-well plate for the absorbance measurement at $570 \mathrm{~nm}$ using a microplate reader. Cells cultured on the plate without nanofibers scaffolds were used as the control group. For cell spreading and attachment examination, cytoskeleton of cells cultured on the nanofiber scaffolds was stained with actin green and nuclei were counter stained by DAPI at $24 \mathrm{~h}$ and 7 -day time points. 


\section{Determination of ALP activity}

The ALP activity of the cells seeded on different scaffolds was analyzed for evaluating the osteoconductive ability of nanofibrous scaffolds. After 7 days of cell seeding, the plates were washed twice with an ice-cold PBS and lysed for freeze-thawing. The supernatant was used for detecting ALP activity by an assay kit according to the manufacturer's instructions.

\section{Mineralized matrix formation assay}

MC3T3-E1 cells were seeded on different scaffolds in 48 -well plates for 21 days. The medium was changed every 3 days with differentiation medium containing $10 \mu \mathrm{M}$ $\beta$-glycerophosphate and $50 \mu \mathrm{g} / \mathrm{mL}$ ascorbic acid. The scaffolds loaded with cells were washed twice with PBS, subsequently fixed with $95 \%$ ethanol for $10 \mathrm{~min}$, and then washed with deionized water and incubated with $0.1 \%$ ARS solution at $37^{\circ} \mathrm{C}$ for $30 \mathrm{~min}$. Then, 10 visual fields were randomly selected for data analysis. Quantification of ARS staining was performed by elution with $10 \%(\mathrm{w} / \mathrm{v})$ cetylpyridinium chloride for $10 \mathrm{~min}$ at room temperature, and the absorbance was measured at $570 \mathrm{~nm}$.

\section{RNA extraction and real-time quantitative polymerase chain reaction assay}

The total cellular RNA was extracted from MC3T3-E1 cells cultured on the scaffolds after 7 days using Trizol reagent for evaluating the levels of gene expression. Specific osteogenic gene expression of osteocalcin (OCN), runt-related transcription factor 2 (Runx-2), and bone morphogenetic protein (BMP) was measured by real-time quantitative polymerase chain reaction (RT-qPCR), and GAPDH was employed as the house keeping gene. All RNA samples showed an $\mathrm{A}_{260} / \mathrm{A}_{280}$ absorbance 21.8 . cDNA was synthesized from $1-3 \mu \mathrm{g}$ of total RNA using Exscript RTase. To quantitate low expression of mRNA levels for OCN, Runx-2, and BMP, RT-qPCR was carried out using specific primers in an ABI Prism 7000 sequence detection system (Thermo Fisher Scientific). The experiments were performed in triplicate.

\section{Statistical analysis}

Data were presented as mean \pm standard deviation (SD) from three independent experiments. Statistical comparison was analyzed by a one-way analysis of variance (ANOVA), followed by Student's $t$-test analysis which was applied for multiple group comparisons. $P$-value $<0.05$ was considered to be statistically significant.

\section{Results and discussion Characterizations of hydroxyapatite nanoparticles and nanofibrous scaffolds}

Recently, bone regeneration has attracted widespread attention in the field of regenerative medicine for the growing demands of bone repair and treatment caused by all sorts of bone injuries or diseases. ${ }^{22}$ Synthetic polymers with structures similar to ECM and type I collagen in bone have been synthesized. ${ }^{23}$ The novel materials mimicked the structure of natural bone and have been shown to be promising potential materials for application in bone engineering. ${ }^{24}$ Electrospun spinning nanofibrous scaffolds provide a suitable surface for cell attachment and proliferation by imitating the structure of natural ECM. ${ }^{25}$ In this study, HA nanoparticles were prepared by sol-gel method; then four nanofiber scaffolds including PLLA, PLLA/HA, PLLA/Coll, and PLLA/Coll/ HA were synthesized using electrospun method. The effects of the four nanofiber scaffolds on the osteogenic behavior were examined using MC3T3-E1cells in vitro. The results showed that PLLA/Coll/HA scaffolds have a significant effect on proliferation, differentiation, and mineralization of MC3T3-E1 cells.

To better evaluate their potential biological effects, nanofiber scaffolds should be characterized appropriately before the biological experiments. The TEM results showed that HA nanoparticles were sphere-like with approximate diameters of $80 \mathrm{~nm}$ (Figure 1A). The crystal phase and structure of HA nanoparticles were measured by powder XRD method. All the diffraction peaks could be indexed to hexagonal crystal phase of hydroxyapatite which belonged to space group P63/m (JCPDS No 01-074-0565). The peaks observed in all diffraction patterns correspond to the (002), (211), (112), (300), (202), (310), (222), (213), and (004) planes, respectively (Figure 1B). No other impurities could be detected. It was also revealed that HA nanoparticles exhibited sharp diffraction peaks, indicating a high crystallinity. The size distribution of HA nanoparticles in the culture medium was investigated using a DLS method, which showed that the average size of HA nanoparticles in the culture medium was $82.9 \pm 24.2 \mathrm{~nm}$ (Figure 1C). The DLS analysis showed that the HA nanoparticles were homogeneously dispersed in culture medium.

The surface morphology and size of nanofibrous scaffolds play an important role in cell attachment and proliferation. Figure 2 shows the SEM micrographs of different electrospun scaffolds. It can be seen that the diameter of PLLA, PLLA/HA, PLLA/Coll, and PLLA/Coll/HA was about 230, 220,300 , and $330 \mathrm{~nm}$, respectively. There was a significant 
A

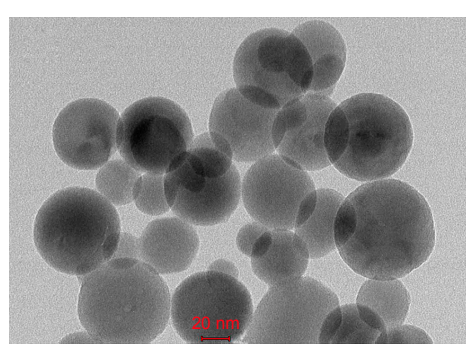

B

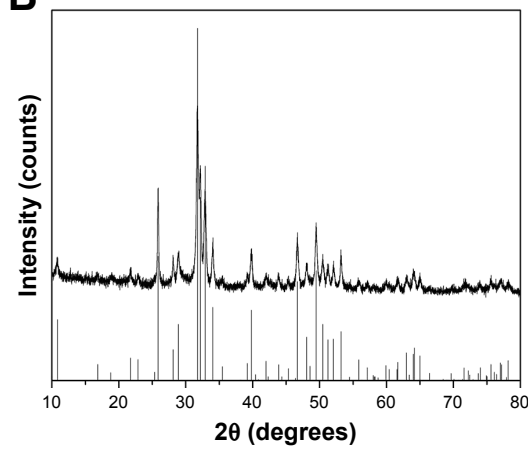

C

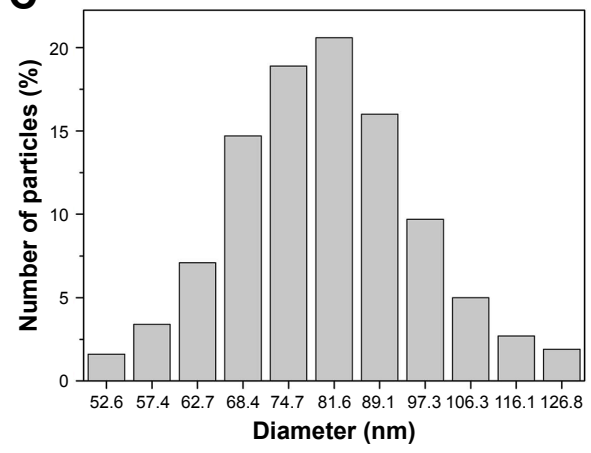

Figure I Characterizations of hydroxyapatite nanoparticles.

Notes: (A) TEM images; (B) XRD patterns; (C) size distribution patterns of hydroxyapatite nanoparticles in culture medium. Abbreviations: TEM, transmission electron microscope; $X R D, X$-ray diffraction.

difference between the diameters of PLLA/HA and PLLA/ Coll/HA nanofibers. However, PLLA/HA nanofiber scaffolds were found to have diameter similar to that of PLLA nanofibers. It indicated that collagen plays an important role in determining the diameter of the electrospun scaffolds. The different size distribution of nanofiber scaffolds possibly attribute to the polarity of materials included in the electrospun scaffolds. ${ }^{26,27}$ The SEM images of nanofibers indicated that PLLA, PLLA/HA, PLLA/Coll, and PLLA/Coll/HA nanofiber scaffolds were composed of ultrafine fibers and interconnected pores. This structure can ideally mimic the ECM and facilitate nutrient transport. ${ }^{28}$ The HA nanoparticles can be seen in the PLLA/HA and PLLA/Coll/HA scaffolds.
The elemental analysis showed that the elemens of $\mathrm{C}, \mathrm{O}$, $\mathrm{Ca}$, and $\mathrm{P}$ were observed in PLLA/HA and PLLA/Coll/HA scaffolds, whereas $\mathrm{Ca}$ and $\mathrm{P}$ were not observed in PLLA and PLLA/Coll scaffolds (Figure 3). The result indicated that HA nanoparticles dispersed in a uniform manner in PLLA/HA and PLLA/Coll/HA scaffolds.

The FTIR results of different nanofibrous scaffolds are shown in Figure 4A. PLLA showed the characteristic carbonyl peak at $1,760 \mathrm{~cm}^{-1}, \mathrm{C}-\mathrm{O}$ stretching at $1,090 \mathrm{~cm}^{-1}$, and $-\mathrm{CH}-$ stretching vibrations at $3,000 \mathrm{~cm}^{-1}$. The characteristic amide 1 and amide 2 peaks of collagen at $1,660 \mathrm{~cm}^{-1}$ and $1,550 \mathrm{~cm}^{-1}$ can be observed in PLLA/Coll and PLLA/Coll/HA nanofibers. The PLLA/HA and PLLA/Coll/HA nanofibers
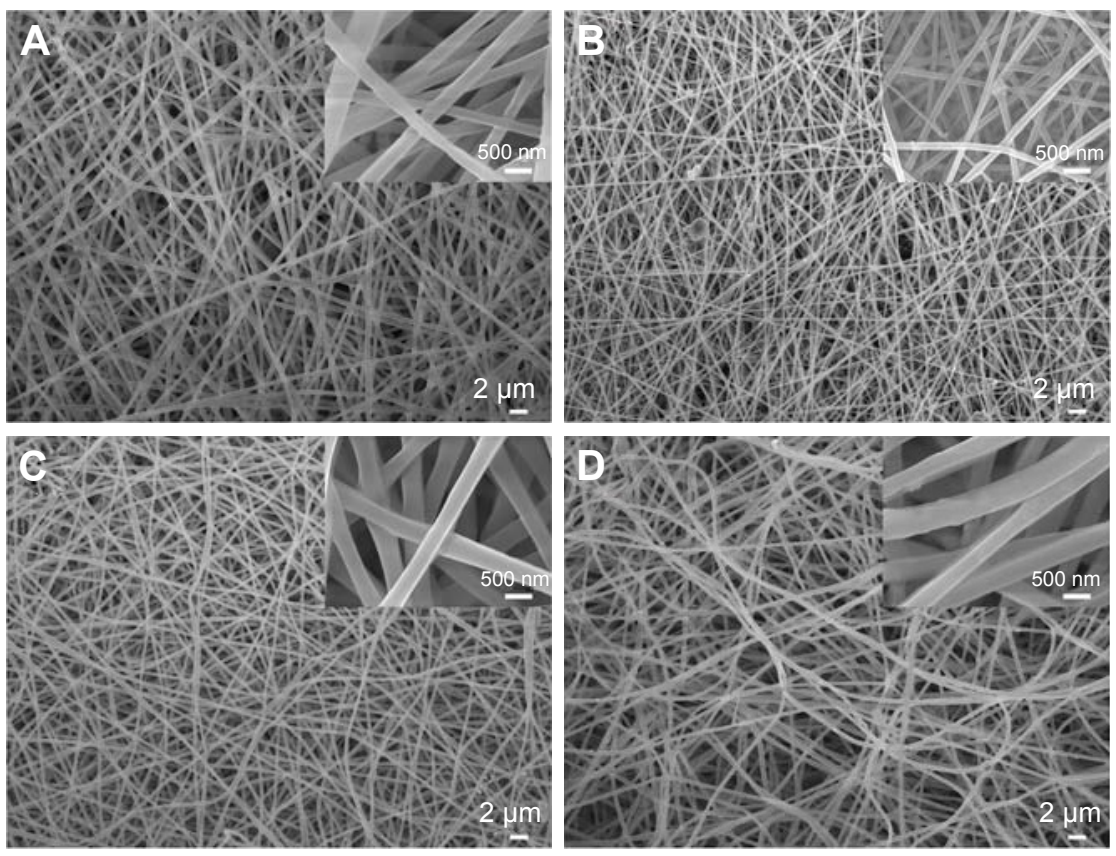

Figure 2 Scanning electron micrographs of different electrospun scaffolds. Notes: (A) PLLA; (B) PLLA/HA; (C) PLLA/Coll; (D) PLLA/Coll/HA.

Abbreviations: PLLA, poly(L-lactide); HA, hydroxyapatite; Coll, collagen. 

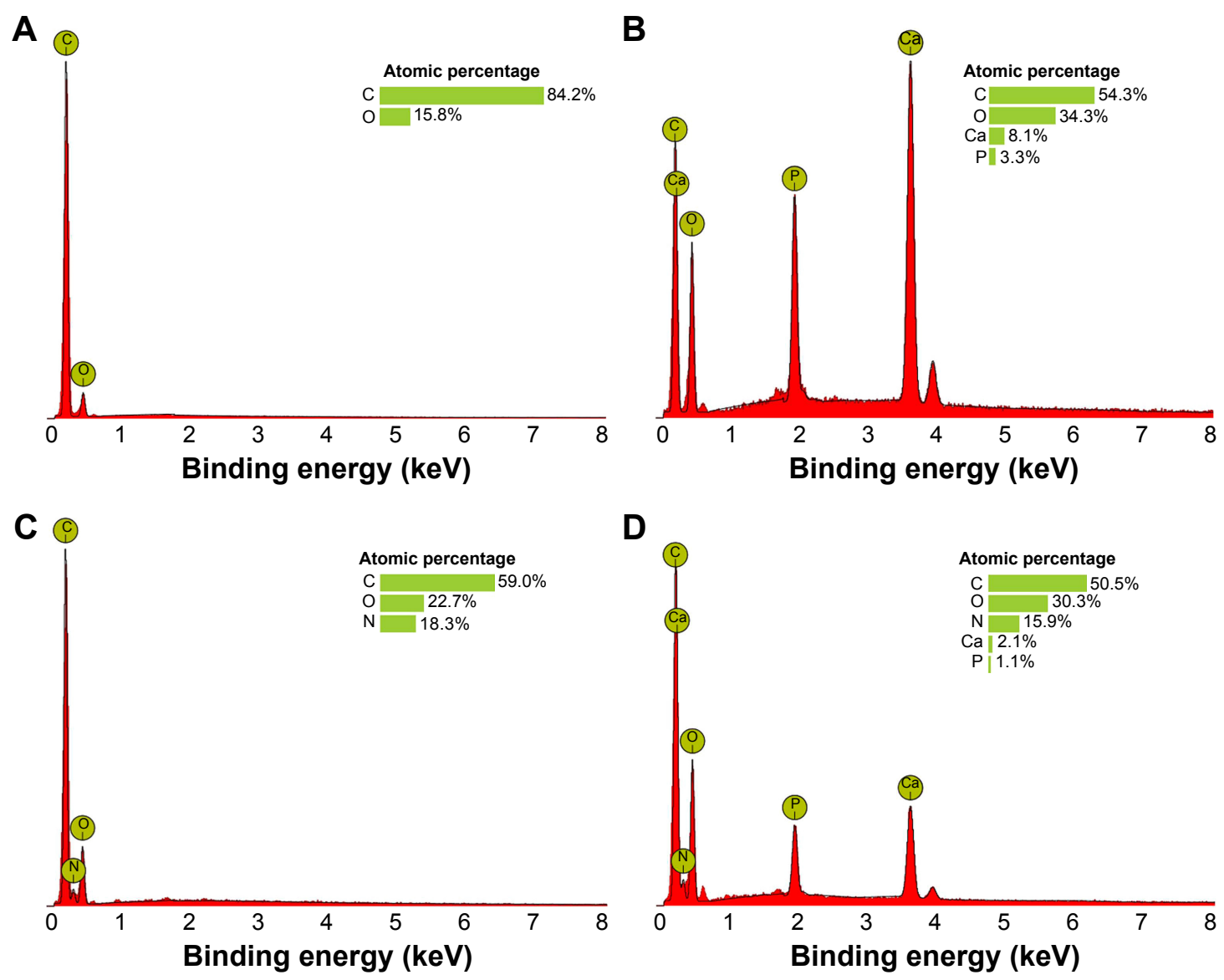

Figure 3 The elements of different electrospun scaffolds analyzed by energy-dispersive $X$-ray spectroscopy. Notes: (A) PLLA; (B) PLLA/HA; (C) PLLA/Coll; (D) PLLA/Coll/HA.

Abbreviations: PLLA, poly(L-lactide); HA, hydroxyapatite; Coll, collagen.

indicated that the characteristic vibrations of the groups of HA occurred at $612 \mathrm{~cm}^{-1}$ and $571 \mathrm{~cm}^{-1}$. Figure 4B shows typical stress-strain curves of different electrospun nanofibrous scaffolds. The average tensile strength of PLLA scaffold was $3.95 \pm 0.16 \mathrm{MPa}$ and has an elongation at break of $17.63 \%$. The PLLA/Coll scaffolds showed a tensile strength of $2.71 \pm 0.15$ MPa. With introduction of HA, the tensile strength of PLLA/ HA and PLLA/Coll/HA scaffolds increased to $3.41 \pm 0.12$ $\mathrm{MPa}$ and $2.75 \pm 0.13 \mathrm{MPa}$, respectively. The tensile strength was higher than that of PLLA/Coll scaffolds. However, the PLLA/Coll/HA scaffolds showed analogous elongation at break of $18.25 \%$ compared to that of PLLA nanofibers. In general, the incorporation of HA nanoparticles in PLLA increased the tensile properties of the electrospun scaffolds. The incorporation of inorganic nanoparticles into organic polymers can improve mechanical properties of the composite scaffolds, such as tensile strength, because HA nanoparticles are hard inorganic components. The increment of tensile strength possibly attributed to an increase in rigidity. These results were in agreement with previous results reported by other researchers. ${ }^{29,30}$ However, the dispersion of collagen slightly decreased the tensile properties of scaffolds. The hydrophilicity of the different scaffolds was examined by static WCA analysis. The PLLA scaffolds had a WCA of $115.2^{\circ}$, which indicated that PLLA was a hydrophobic material. After mixing with collagen, the WCA of the PLLA/ Coll scaffolds decreased to $79.1^{\circ}$. However, the WCA of the PLLA/HA scaffolds increased to $118.7^{\circ}$ after HA nanoparticles were introduced in the composite scaffolds. The WCA of the PLLA/Coll/HA scaffolds decreased to $102.1^{\circ}$ compared with that of PLLA/HA scaffolds (Figure 4C). The results indicated that the hydrophility of the scaffolds can be improved by collagen and reduced by HA. The nanofibrous scaffolds synthesized by electrospinning method had sustained WCA values, and there was no infiltration leakage within $1 \mathrm{~min}$ (Figure 4D). The results demonstrated that the several electrospun scaffolds synthesized demonstrated favorable stability with respect to hydrophilicity.

\section{In vitro degradation}

An ideal bone repair scaffold material should degrade during 2 months of bone repair progress..$^{31}$ Thus, the degradability 
A

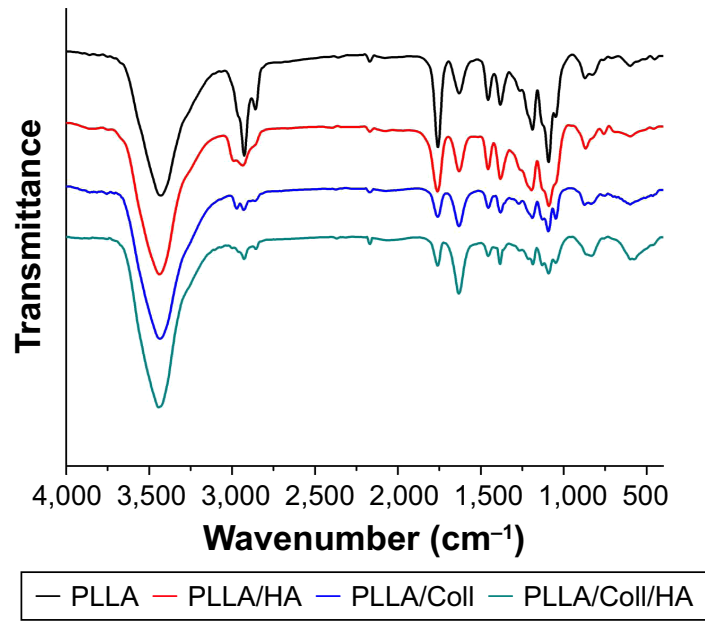

C

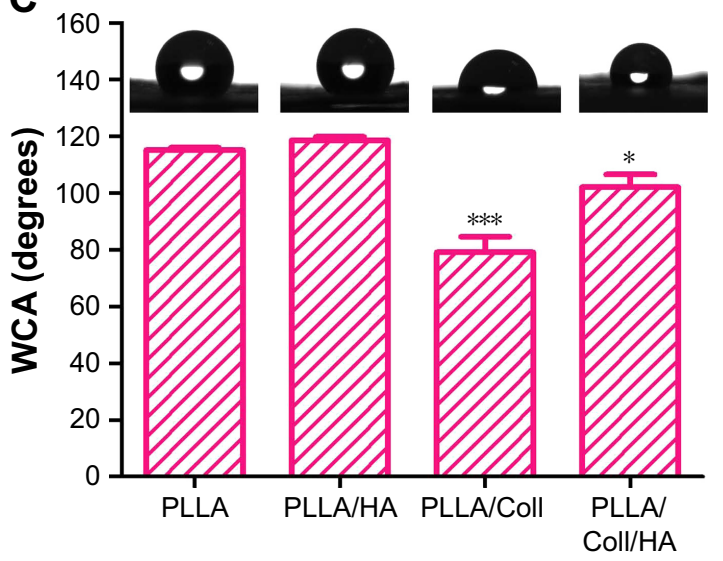

B
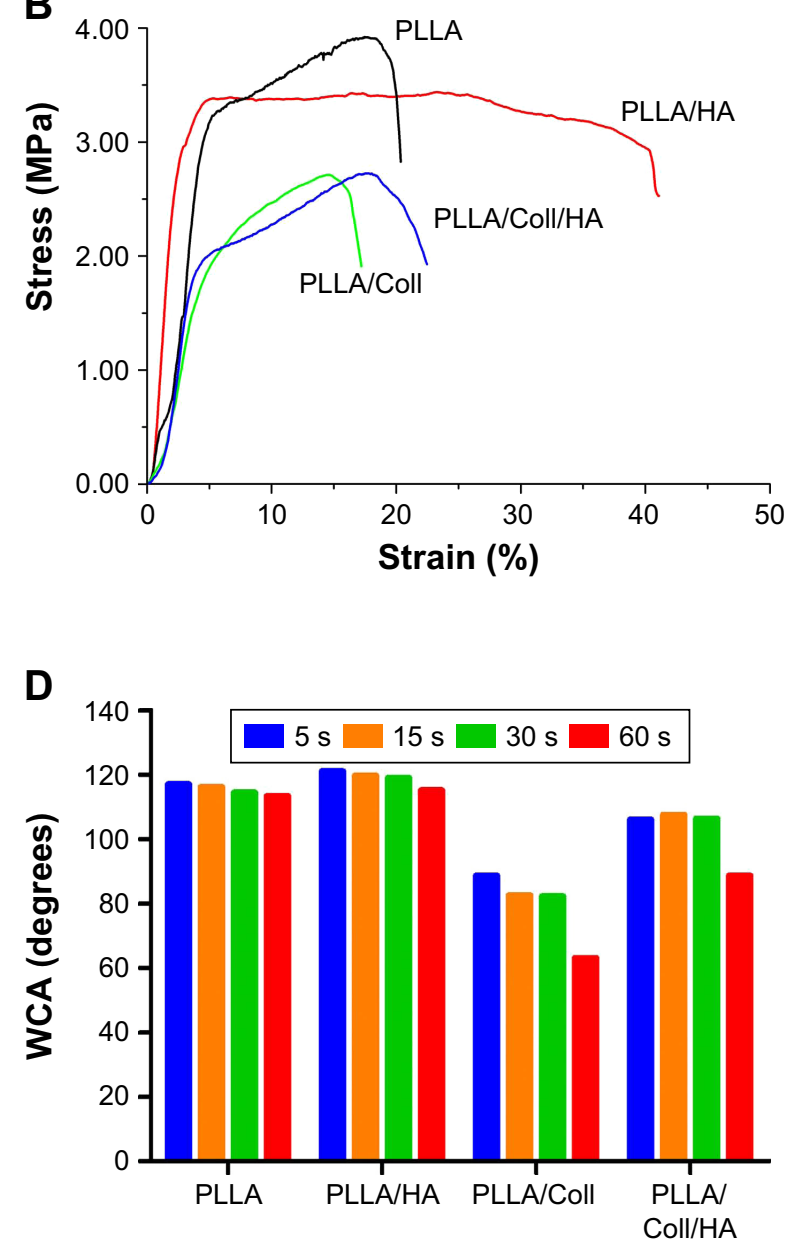

Figure 4 The Fourier transform infrared spectroscopy, contact angle, and tensile measurements of different electrospun scaffolds.

Notes: (A) FTIR spectra; (B) tensile properties; (C) averaged water contact angles (the shapes above the bars are the images captured after the water droplet dripped on the surface of composite fibrous scaffolds for $15 \mathrm{~s}$ ); (D) water contact angles measured at 5, 15, 30, and $60 \mathrm{~s}$. Data are expressed as mean \pm standard deviation from three independent experiments. $* P<0.05$, $* * * P<0.001$ compared with the PLLA group.

Abbreviations: PLLA, poly(L-lactide); HA, hydroxyapatite; Coll, collagen; FTIR, Fourier transform infrared; WCA, water contact angle.

of different scaffolds in SBF at 10, 30, 50, and 80 days was examined. The morphology of different scaffolds after degradation was observed using SEM (Figure 5). All the scaffolds had a swollen state with a rough surface compared with 0 days due to the long-term immersion in SBF. PLLA/HA scaffolds became thinner gradually and had a rough surface due to the PLLA degradation, and HA particles appeared from the surface of scaffolds. PLLA/Coll scaffolds showed a broken morphology at 80 days because of the collagen degradation. PLLA/Coll/HA scaffolds showed a swollen morphology at 30 and 50 days but showed a broken morphology at 80 days because of the collagen degradation, in which more HA nanoparticles can be found on scaffolds. The in vitro scaffold degradation was studied by monitoring the weight loss up to 80 days (Figure 6). PLLA/Coll/HA, PLLA/Coll, and PLLA/HA scaffolds had 34.5\% $04.2 \%$, $68.2 \% \pm 6.5 \%$, and $16.8 \% \pm 1.3 \%$ weight loss after 80 days of immersion in SBF, respectively. PLLA/Coll/HA scaffolds demonstrated moderate weight loss levels depending on their composition.

\section{Effects of electrospun scaffolds on cellular viability}

The biocompatibility of the scaffolds is closely related to cell attachment, adhesion, and spreading when in contact with them. The quality of initial attachment, adhesion, and spreading of cells on the scaffolds will influence the proliferation capacity of cells as well as directional differentiation on them. ${ }^{32,33}$ The cell viability on the scaffolds at 1,3 , and 7 days is shown in Figure 7A. The results indicated that there was no significant difference in cell viability among four scaffolds at 1 and 3 days. However, the cell viability at day 7 was higher than that at days 1 and 3. After cells were cultured on PLLA, PLLA/HA, PLLA/Coll, and PLLA/ Coll/HA for 7 days, cell viability was increased to $105.5 \%$, $111.1 \%, 114.4 \%$, and $112.6 \%$, respectively, compared to 

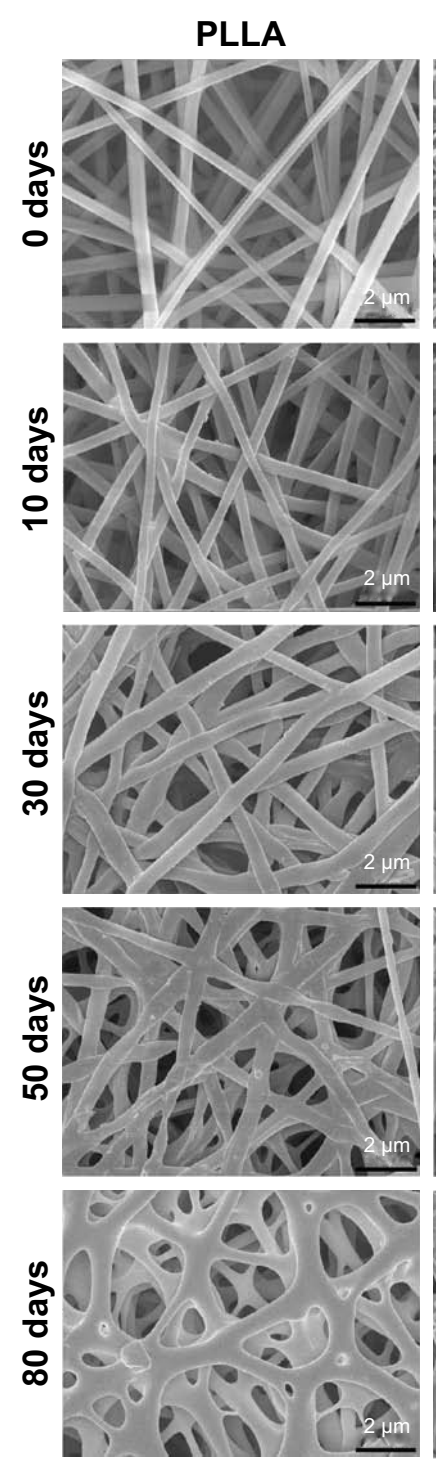

Figure 5 The morphology at different time points of various scaffolds after degradation. Abbreviations: PLLA, poly(L-lactide); HA, hydroxyapatite; Coll, collagen.

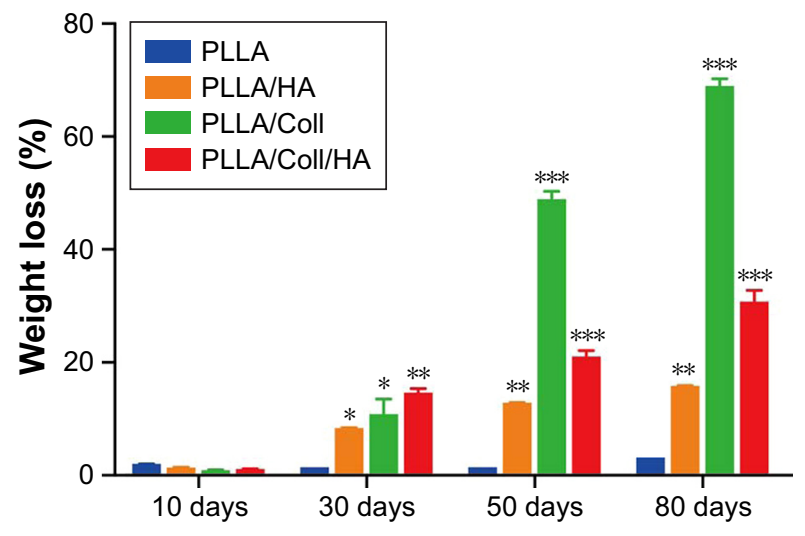

Figure 6 Weight losses of various scaffolds in vitro at $37^{\circ} \mathrm{C}$ in simulated body fluid at $10,30,50$, and 80 days.

Notes: Data are expressed as mean \pm standard deviation from three independent experiments. $* P<0.05$, $* * P<0.01$, $* * * P<0.001$ compared with the PLLA group. Abbreviations: PLLA, poly(L-lactide); HA, hydroxyapatite; Coll, collagen. the control. The results showed that the scaffolds exhibited a good biocompatibility toward MC3T3-E1 cells and were conducive to cell growth. Furthermore, the morphology of cells attached on scaffolds was inspected using fluorescence microscope. Fluorescence microscopy images indicated that the surface of these scaffolds was more conducive to cell adhesion. The cells protruded more filopodia and lamellipodia and stretched better on the nanofiber scaffolds than on the plate. At day 1, the cells on the PLLA/Coll and PLLA/Coll/HA scaffolds had spread out and possessed much longer filopodia compared to other scaffolds. It was found that the adhered MC3T3-E1 cells stretched better on the PLLA/Coll and PLLA/Coll/HA scaffolds than the control group at day 7 (Figure 7B). The promotion effects of the 
A

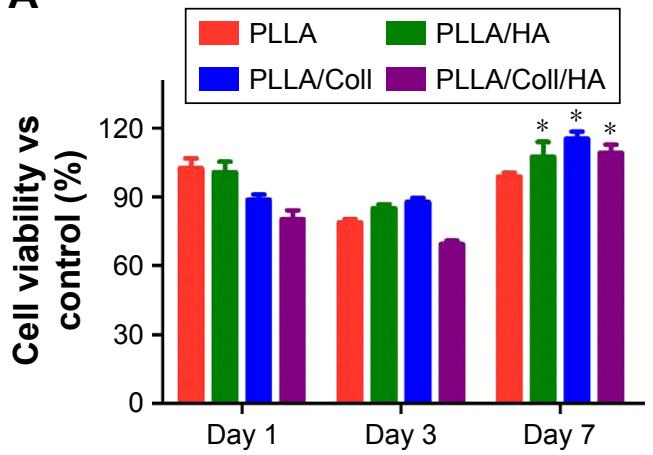

C

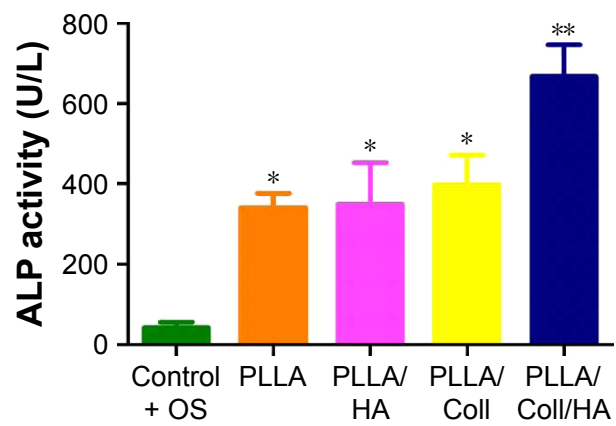

B

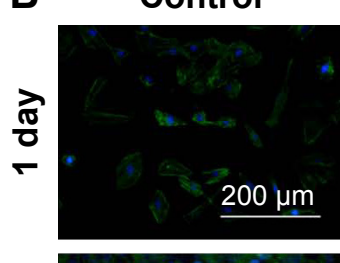

PLLA
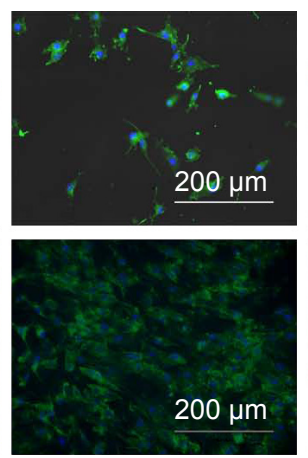

PLLA/HA
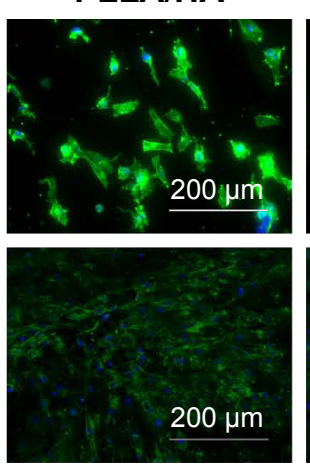

PLLA/Coll
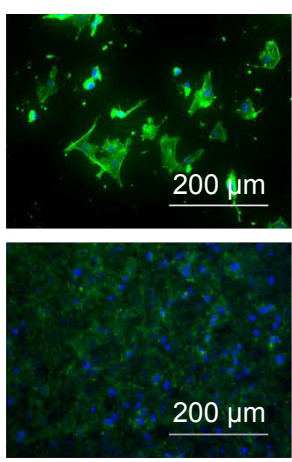

PLLA/Coll/HA
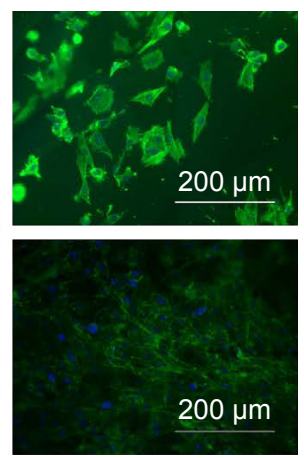

Figure 7 The effects of different electrospun scaffolds on the viability and ALP activity of MC3T3-EI cells.

Notes: (A) The viability of cells cultured on different electrospun scaffolds for I, 3, and 7 days; (B) fluorescence microscopy of cells cultured on different electrospun scaffolds for I and 7 days; (C) ALP activity of cells cultured on different electrospun scaffolds. The blue dots indicate the DNA of live cells stained with DAPI. The green color indicates the cytoskeleton of cells stained with actin green. Data are expressed as mean \pm standard deviation from three independent experiments $(n=6)$. $* * P<0.0$ I, $* P<0.05$ compared with the corresponding control group.

Abbreviations: PLLA, poly(L-lactide); HA, hydroxyapatite; Coll, collagen; ALP, alkaline phosphatase; OS, osteogenic inducing supplements; DAPI, 4',6-diamidino-2-phenylindole.

PLLA/Coll and PLLA/Coll/HA scaffolds were higher than those of PLLA scaffolds at day 7, which agreed with the results of MTT. The introduction of collagen in the scaffolds can enhance cellular adherence, attachment, and spreading, which agreed with the previous study. ${ }^{34}$ The incorporation of collagen and HA into PLLA electrospun nanofibers could mimic the component and nanotopography of bone to some extent. ${ }^{35,36}$ Thus, the PLLA/Coll/HA scaffolds were more favorable for bone cell adhesion.

\section{Effects of electrospun scaffolds on cellular differentiation}

The effects of the scaffolds on cellular differentiation were determined by ALP, qPCR, and alizarin red staining assays. As an early marker of osteogenic differentiation, ALP plays a major role in the formation of mineral deposits in the matrix during new bone formation. ${ }^{37,38}$ The ALP activity of MC3T3-E1 cells cultured on four various scaffolds is shown in Figure 7C. After 7 days of cell culture, the ALP activity of cells on nanofiber scaffolds was significantly higher than that of the control group. Meantime, the ALP activity of cells on PLLA/HA and PLLA/Coll nanofiber scaffolds was increased by $2 \%$ and $16 \%$, respectively, than that on PLLA scaffolds. Moreover, the ALP activity of cells on PLLA/ Coll/HA nanofiber scaffolds increased by $95 \%, 91 \%$, and $68 \%$ higher than that on PLLA, PLLA/HA, and PLLA/Coll nanofibers scaffolds, respectively. The results indicated that the nanofiber scaffolds can promote the differentiation of MC3T3-E1 cells. The promotion effects of PLLA/Coll/HA composite scaffolds were more significant than those demonstrated by other fiber scaffolds. Meantime, the results of ALP activity showed the significance of HA and Coll in bone regeneration, where the presence of HA and Coll in PLLA/ Coll/HA caused a stimulation of bone cell response.

\section{Effects of electrospun scaffolds on cellular mineralization}

In the early stages of osteogenic differentiation, the level of ALP activity would increase, and mineral production is a later marker of osteogenic differentiation. Mineralization function 
is a necessary condition of osteoblasts to form bone calcification eventually. ${ }^{39}$ Mineralization of MC3T3-E1 cells cultured on different scaffolds was determined both qualitatively and quantitatively using ARS staining method after 21 days of culture. The mineralized matrix nodules of cells cultured on different nanofiber scaffolds stained by ARS are shown in Figure 8. There were less mineral deposits on plate and PLLA scaffolds, whereas more calcium deposits were stained by ARS on PLLA/HA and PLLA/Coll/HA scaffolds. With the introduction of HA nanoparticles, more calcium deposition can be detected on the nanofiber scaffolds. The cells cultured on PLLA/Coll/HA scaffolds clearly showed more calcium deposition than those cultured on other scaffolds. The quantitative evaluation of calcium deposition also proved that MC3T3-E1 mineralization on PLLA/Coll/HA nanofiber scaffolds was significantly higher than that on other scaffolds (Figure 8). That is, the composite scaffolds of PLLA/ Coll/HA were optimal for inducing the mineralization of MC3T3-E1 cells. Early studies demonstrated that millimolar concentrations of calcium ions could enhance osteogenic differentiation. ${ }^{40}$ Thus, calcium ions released from HA may enhance osteogenic differentiation. Besides, a better initial event can also lead to a series of preferable cell responses, including cell growth, division, and differentiation. The osteoblasts begin to secrete mineral matrix, and mineralization is a marker of maturation. Enhanced calcium binding and matrix deposition were observed in the PLLA/HA and PLLA/Coll/HA composite nanofibrous scaffolds due to the HA incorporation. Furthermore, the addition of Coll significantly enhanced the mineralization function.
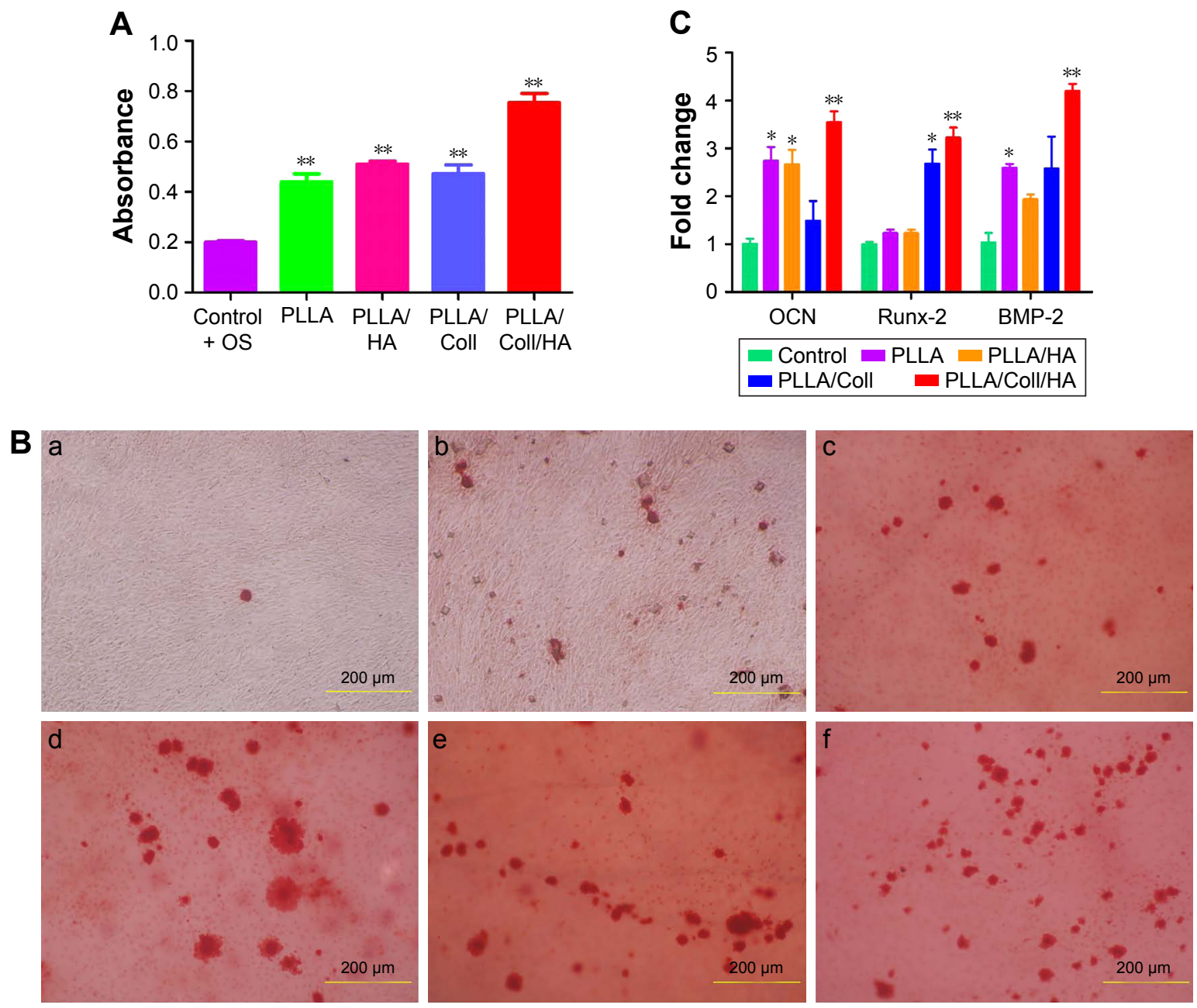

Figure 8 The effects of different electrospun scaffolds on the cell differentiation of MC3T3-EI cells.

Notes: (A) Quantification of the mineralized matrix of cells cultured on different nanofiber scaffolds; (B) the mineralized matrix nodules of cells cultured on different nanofiber scaffolds stained by ARS: (a) control without differentiation medium, (b) control with differentiation medium, (c) PLLA, (d) PLLA/HA, (e) PLLA/Coll, (f) PLLA/Coll/HA; (C) the expression of mRNA for OCN, Runx-2, and BMP-2 in cells cultured on different nanofiber scaffolds. Data are expressed as mean \pm standard deviation from three independent experiments $(\mathrm{n}=6)$. $* * P<0.0 \mathrm{I}, * \mathrm{P}<0.05$ compared with the corresponding control group.

Abbreviations: PLLA, poly(L-lactide); HA, hydroxyapatite; Coll, collagen; ARS, alizarin red-S; OS, osteogenic inducing supplements. 
Table I RT-qPCR primer sequence

\begin{tabular}{lll}
\hline $\begin{array}{l}\text { Target } \\
\text { gene }\end{array}$ & $\begin{array}{l}\text { Primer } \\
\text { sequence }\left(\mathbf{5}^{\prime} \rightarrow \mathbf{3}^{\prime}\right)\end{array}$ & $\begin{array}{l}\text { Anti-primer } \\
\text { sequence }\left(\mathbf{3}^{\prime} \rightarrow \mathbf{5}^{\prime}\right)\end{array}$ \\
\hline OCN & AACATGACCAAAAA & CATTGTTTCCTGTG \\
& CCAAAAGTG & TCTTCTGG \\
Runx-2 & TTCTCCAACCCACG & CAGGTACGTGTGGT \\
& AATGCAC & AGTGAGT \\
BMP-2 & TGGCCCATTTAGAG & AGGCATGATAGCCC \\
& GAGAACC & GGAGG \\
GAPDH & GACTTCAACAGCAA & TCCACCACCCTGTT \\
& CTCCCAC & GCTGTA \\
\hline
\end{tabular}

Abbreviation: RT-qPCR, real-time quantitative polymerase chain reaction.

The expression of OCN, Runx-2, and BMP-2 plays an important role in the progress of osteogenic differentiation. ${ }^{41}$ To further examine the effects of nanofiber scaffolds on osteogenic differentiation of cells, the gene expression of MC3T3-E1 cells was examined. The primer sequences are summarized in Table 1. The expression levels of OCN, Runx-2, and BMP-2 are shown in Figure 8C. The results showed that the mRNA levels of OCN, Runx-2, and BMP-2 were significantly increased after 14 days of treatment with different nanofiber scaffolds, compared with the control group. Moreover, the gene expression of cells cultured on PLLA/Coll/HA nanofiber scaffolds was higher than that on the other three nanofiber scaffolds. It means that the PLLA/ Coll/HA nanofiber scaffolds can upregulate the expression of OCN, Runx-2, and BMP-2 more significantly. Higher expression of osteogenic genes in PLLA/Coll/HA group demonstrated that the incorporation of Coll and HA into PLLA scaffolds could efficiently promote the differentiation of MC3T3-E1 cells. These results demonstrated that PLLA/Coll/ HA composite scaffolds had better osteoinductive activity in vitro. Thus, the PLLA/Coll/HA composite scaffolds have potential applications in bone tissue engineering.

\section{Conclusion}

In summary, several biocomposite nanofiber scaffolds including PLLA, PLLA/HA, PLLA/Coll, and PLLA/Coll/HA were prepared by electrospinning method and characterized by SEM, EDS, FTIR, stress-strain curves, and hydrophilic property. Then, the biodegradability and osteoinductive properties of the nanofiber scaffolds were evaluated. The results indicated that the PLLA/Coll/HA scaffolds had better biodegradability, cell proliferation, and osteoinductive activity than other scaffolds. The combination of PLLA, collagen, and HA mimics the nanoscale structure of bone, thus supporting the fact that the scaffolding materials have potential application for bone regeneration in tissue engineering.

\section{Acknowledgments}

This work was supported by the National Natural Science Foundation of China (21471044), the Joint Funds of the National Natural Science Foundation of China (U1732127), the Natural Science Foundation of Hebei Province (B2017201135), the Youth Talent Fund Project of Hebei Education Department (BJ2014007), Challenge Cup National College Student Curricular Academic Science and Technology Works Competition, and the College Students' Innovative Training Project of Hebei University (2016053).

\section{Disclosure}

The authors report no conflicts of interest in this work.

\section{References}

1. Noori A, Ashrafi SJ, Vaez-Ghaemi R, et al. A review of fibrin and fibrin composites for bone tissue engineering. Int J Nanomed. 2017;12: 4937-4961.

2. Prabhakaran MP, Venugopal J, Ramakrishna S. Electrospun nanostructured scaffolds for bone tissue engineering. Acta Biomater. 2009; 5(8):2884-2893.

3. Sahoo NG, Pan YZ, Li L, He CB. Nanocomposites for bone tissue regeneration. Nanomedicine. 2013;8(4):639-653.

4. Xie CM, Lu X, Wang KF, et al. Silver nanoparticles and growth factors incorporated hydroxyapatite coatings on metallic implant surfaces for enhancement of osteoinductivity and antibacterial properties. ACS Appl Mater Interfaces. 2014;6(11):8580-8589.

5. Diomede F, Zini N, Gatta V, et al. Human periodontal ligament stem cells cultured onto cortico-cancellous scaffold drive bone regenerative process. Eur Cell Mater. 2016;32:181-201.

6. Zhang Y, Ye L, Cui J, et al. A biomimetic poly(vinyl alcohol)carrageenan composite scaffold with oriented microarchitecture. ACS Biomater Sci Eng. 2016;2(4):544-557.

7. Kim TG, Shin H, Lim DW. Biomimetic scaffolds for tissue engineering. Adv Funct Mater. 2012;22(12):2446-2468.

8. Pina S, Oliveira JM, Reis RL. Natural based nanocomposites for bone tissue engineering and regenerative medicine. Adv Mater. 2015; 27(7):1143-1169.

9. Cui W, Zhou Y, Chang J. Electrospun nanofibrous materials for tissue engineering and drug delivery. Sci Technol Adv Mat. 2010;11(1): 1468-6996.

10. Agarwal S, Wendorff J H, Greiner A. Progress in the field of electrospinning for tissue engineering applications. Adv Mater. 2009;21(32-33): 3343-3351.

11. Ravarian R, Zhong X, Barbeck M, et al. Nanoscale chemical interaction enhances the physical properties of bioglass composites. ACS Nano. 2013;7(10):8469-8483.

12. Siqueira IA, Corat MA, Bd C, et al. In vitro and in vivo studies of novel poly(D,L-lactic acid), superhydrophilic carbon nanotubes, and nanohydroxyapatite scaffolds for bone regeneration. ACS Appl Mater Interfaces. 2015;7(18):9385-9398.

13. Sachot N, Roguska A, Planell JA, et al. Fast-degrading PLA/ ORMOGLASS fibrous composite scaffold leads to a calcium-rich angiogenic environment. Int J Nanomed. 2017;12:4901-4919.

14. Wang JB, Wu DY, Zhang ZZ, et al. Biomimetically ornamented rapid prototyping fabrication of an apatite-collagen-polycaprolactone composite construct with nano-micro-macro hierarchical structure for large bone defect treatment. ACS Appl Mater Interfaces. 2015;7(47):26244-26256.

15. Yang F, Both S K, Yang X, et al. Development of an electrospun nanoapatite/PCL composite membrane for GTR/GBR application. Acta Biomater. 2009;5(9):3295-3304. 
16. Andersson SR, Hakkarainen M, Inkinen S. Polylactide stereocomplexation leads to higher hydrolytic stability but more acidic hydrolysis product pattern. Biomacromolecules. 2010;11(4):1067-1073.

17. Martínezvázquez FJ, Cabañas MV, Paris JL, et al. Fabrication of novel Si-doped hydroxyapatite/ gelatine scaffolds by rapid prototyping for drug delivery and bone regeneration. Acta Biomater. 2015;15:200-209.

18. Qi H, Ye Z, Ren H, et al. Bioactivity assessment of PLLA/PCL/HAP electrospun nanofibrous scaffolds for bone tissue engineering. Life Sci. 2016;148:139-144.

19. Liao GY, Jiang SB, Xia H, et al. Preparation and characterization of aligned PLLA/PCL/HA composite fibrous membranes. J Macromol Sci A. 2012;49(11):946-951.

20. Anugraha G, Madhumathi J, Prita PJJ, et al. Biodegradable poly-l-lactide based microparticles as controlled release delivery system for filarial vaccine candidate antigens. Eur J Pharmacol. 2015;747:174-180.

21. Heinemann S, Heinemann C, Jäger M, et al. Effect of silica and hydroxyapatite mineralization on the mechanical properties and the biocompatibility of nanocomposite collagen scaffolds. ACS Appl Mater Interfaces. 2011;3(11):4323-4331.

22. Fisher MB, Mauck RL. Tissue engineering and regenerative medicine: recent innovations and the transition to translation. Tissue Eng Part B Rev. 2013;19(1):1-13.

23. Wolf MT, Dearth CL, Sonnenberg SB, et al. Naturally derived and synthetic scaffolds for skeletal muscle reconstruction. Adv Drug Deliver Rev. 2015;84:208-221.

24. Zhou Y, Yao H, Wang J, et al. Greener synthesis of electrospun collagen/ hydroxyapatite composite fibers with an excellent microstructure for bone tissue engineering. Int J Nanomed. 2015;10:3203-3215.

25. Yoshimoto H, Shin Y M, Terai H, et al. A biodegradable nanofiber scaffold by electrospinning and its potential for bone tissue engineering. Biomaterials. 2003;24(12):2077-2082.

26. Park K, Kang H, Lee S, et al. Biomimetic nanofibrous scaffolds: preparation and characterization of $\mathrm{PGA} /$ chitin blend nanofibers. Biomacromolecules. 2006;7:635-643.

27. Badami AS, Kreke MR, Thompson MS, et al. Effect of fiber diameter on spreading, proliferation and differentiation of osteoblastic cells on electrospun poly (lactic acid) substrates. Biomaterials. 2006;27:596-606.

28. Peng S, Jin G, Li L, et al. Multi-functional electrospun nanofibres for advances in tissue regeneration, energy conversion and storage, and water treatment. Chem Soc Rev. 2016;45(5):1225-1241.

29. Mehrasa M, Asadollahi MA, Nasri-Nasrabadi B, et al. Incorporation of mesoporous silica nanoparticles into random electrospun PLGA and PLGA/gelatin nanofibrous scaffolds enhances mechanical and cell proliferation properties. Mater Sci Eng C Mater Biol Appl. 2016; $66: 25-32$.
30. Mehrasa M, Anarkoli AO, Rafienia M, et al. Incorporation of zeolite and silica nanoparticles into electrospun PVA/collagen nanofibrous scaffolds: the influence on the physical, chemical properties and cell behavior. Int J Polym Mater. 2016;65(9):457-465.

31. Chung JJ, Fujita Y, Li S, et al. Biodegradable inorganic-organic hybrids of methacrylate star polymers for bone regeneration. Acta Biomater. 2017;54:411-418

32. An G, Zhang WB, Ma DK, et al. Influence of VEGF/BMP-2 on the proliferation and osteogenetic differentiation of rat bone mesenchymal stem cells on PLGA/gelatin composite scaffold. Eur Rev Med Pharmacol Sci. 2017;21(10):2316-2328.

33. Asghari Sana F, Çapkın Yurtsever M, Kaynak Bayrak G, et al. Spreading, proliferation and differentiation of human dental pulp stem cells on chitosan scaffolds immobilized with RGD or fibronectin. Cytotechnology. 2017;69(4):617-630.

34. Ngiam M, Liao S, Patil AJ, et al. The fabrication of nano-hydroxyapatite on PLGA and PLGA/collagen nanofibrous composite scaffolds and their effects in osteoblastic behavior for bone tissue engineering. Bone. 2009;45(1):4-16.

35. Bhowmick A, Mitra T, Gnanamani A, et al. Development of biomimetic nanocomposites as bone extracellular matrix for human osteoblastic cells. Carbohydr Polym. 2016;141:82-91.

36. Liao S, Nguyen LT, Ngiam M, et al. Biomimetic nanocomposites to control osteogenic differentiation of human mesenchymal stem cells. Adv Healthc Mater. 2014;3(5):737-751.

37. Ding ZZ, Fan ZH, Huang XW, et al. Bioactive natural proteinhydroxyapatite nanocarriers for optimizing osteogenic differentiation of mesenchymal stem cells. J Mater Chem B. 2016;4(20):3555-3561.

38. Favarin BZ, Andrade MAR, Bolean M, et al. Effect of the presence of cholesterol in the interfacial microenvironment on the modulation of the alkaline phosphatase activity during in vitro mineralization. Colloids Surf B Biointerfaces. 2017;155:466-476.

39. Zhang $\mathrm{S}$, Chen $\mathrm{X}, \mathrm{Hu} \mathrm{Y}$, et al. All-trans retinoic acid modulates Wnt3A-induced osteogenic differentiation of mesenchymal stem cells via activating the PI3K/AKT/GSK3 $\beta$ signalling pathway. Mol Cell Endocrinol. 2016;422:243-253.

40. Barradas AM, Fernandes HA, Groen N, et al. A calcium-induced signaling cascade leading to osteogenic differentiation of human bone marrow-derived mesenchymal stromal cells. Biomaterials. 2012;33(11): 3205-3215.

41. Diomede F, Merciaro I, Martinotti S, et al. miR-2861 is involved in osteogenic commitment of human periodontal ligament stem cells grown onto 3D scaffold. J Biol Regul Homeost Agents. 2016;30(4):1009-1018.
International Journal of Nanomedicine

\section{Publish your work in this journal}

The International Journal of Nanomedicine is an international, peerreviewed journal focusing on the application of nanotechnology in diagnostics, therapeutics, and drug delivery systems throughout the biomedical field. This journal is indexed on PubMed Central, MedLine, CAS, SciSearch $®$, Current Contents ${ }^{\circledR} /$ Clinical Medicine,
Dovepress

Journal Citation Reports/Science Edition, EMBase, Scopus and the Elsevier Bibliographic databases. The manuscript management system is completely online and includes a very quick and fair peer-review system, which is all easy to use. Visit http://www.dovepress.com/ testimonials.php to read real quotes from published authors. 